\title{
OCORRÊNCIA DE PEIXES NÃO-NATIVOS NO SISTEMA ESTUARINO- LAGUNAR DE TRAMANDAÍ, RIO GRANDE DO SUL, BRASIL
}

\author{
Rodrigo Machado ${ }^{1,2}$, Renato B. Dala-Corte ${ }^{3}$, Pedro Carvalho-Neto ${ }^{2}$, Eveline Bernardes Silva ${ }^{4}$, Paulo Henrique Ott ${ }^{5}$ \\ ${ }^{1}$ Grupo de Estudos de Mamíferos Aquáticos do Rio Grande do Sul, Rua Machado de Assis, 1456, Sulbrasileiro, CEP: 95520-000, Osório, RS, \\ Brasil. Fone: 51 3663-9455. E-mail : ecomachado@gmail.com \\ ${ }^{2}$ Universidade Federal do Rio Grande do Sul, Programa de Pós-Graduação em Biologia Animal, Av. Bento Gonçalves, 9500, Bloco IV, Prédio \\ 43435, Sala 123, Agronomia, CEP: 91501-970, Porto Alegre, RS, Brasil. Fone: 5133087696 E-mail: carvalhonetop@ gmail.com \\ ${ }^{3}$ Universidade Federal do Rio Grande do Sul (UFRGS), Programa de Pós-Graduação em Ecologia, Av. Bento Gonçalves, 9500, Agronomia, \\ CEP: 91501-970, Porto Alegre, RS, Brasil. Fone: 5133087623 E-mail: renatocorte@ gmail.com \\ ${ }^{4}$ Centro de Ensino Médio Pastor Dohms, Rua Ararigbóia, 284, Centro, CEP: 95555-000, Capão da Canoa, RS, Brasil. Fone: 51 36653066 \\ E-mail: evelinebernardes@yahoo.com.br \\ ${ }^{5}$ Universidade Estadual do Rio Grande do Sul (UERGS). Laboratório de Ecologia e Conservação de Organismos e Ambientes Aquáticos \\ (ECOAqua). Rua Machado de Assis, 1456, Sulbrasileiro, CEP: 95520-000, Osório, RS, Brasil. Fone: 51 3663-9455. \\ E-mail: paulo.henrique.ott@gmail.com
}

Resumo- O presente trabalho tem como objetivo relatar novos registros de peixes não-nativos no sistema estuarino-lagunar do rio Tramandaí (SELT) e apresentar uma revisão das espécies de peixes invasoras na bacia hidrográfica do rio Tramandaí (BHRT), no litoral norte do Rio Grande do Sul, sul do Brasil. A ocorrência das espécies foi documentada a partir de fotografias fornecidas por pescadores locais obtidas entre 2007 e 2008, além da compilação de trabalhos científicos existentes na literatura. As espécies registradas para o SELT foram: carpacapim (Ctenopharyngodon idella), carpa-húngara (Cyprinus carpio), carpa-cabeça-grande (Hypophthalmichthys nobilis), carpa-prateada (H. molitrix) e uma espécie de Serrasalmidae (cf. Piaractus mesopotamicus). Para a BHRT são ainda relatadas na literatura: peixe-cachorro (Acestrorhynchus pantaneiro), piava (Leporinus obtusidens), tilápia-do-Nilo (Oreochromis niloticus) e porrudo (Trachelyopterus lucenai). A ocorrência dessas espécies na BHRT provavelmente está associada a escapes acidentais ocorridos de tanques de piscicultura nos municípios vizinhos e também aos alagamentos dos campos de cultivo de arroz durante a época de chuvas. A introdução dessas espécies não-nativas representa uma ameaça potencial aos ecossistemas aquáticos de água doce da região, podendo causar sérios danos à diversidade local e à pesca artesanal. Em vista disso, é fundamental que seja realizado um completo mapeamento dos empreendimentos de piscicultura existentes na BHRT e que estas atividades sejam regularizadas e fiscalizadas. Com intuito de incentivar o cultivo de espécies nativas e reduzir as chances de introdução de espécies invasoras é importante também ampliar as atividades de assistência técnica aos piscicultores da região.

Palavras-chave: Espécies não-nativas. Alóctones. Exóticas. Invasoras. Bacia Hidrográfica do Rio Tramandaí.

Abstract - The present work aims to report new records of non-native fishes in the lagoon-estuarine system of Tramandaí (SELT) and review the available information on invasive fish species in the Tramandai river drainage basin (BHRT), in the northern coast of Rio Grande do Sul, southern Brazil. The occurrence of these species was documented from photographs supplied by local fishermen, between 2007 and 2008, as well as from a literature review. The species recorded in the system were: bighead carp (Hypophthalmichthys nobilis), silver carp (H. molitrix), grass carp (Ctenopharyngodon idella), common carp (Cyprinus carpio) and one Serrasalmidae species (cf. Piaractus mesopotamicus). For the BHRT the following species are also reported in the literature: "peixe-cachorro" (Acestrorhynchus pantaneiro), "piava" (Leporinus obtusidens), Nile tilapia (Oreochromis niloticus) and "porrudo" (Trachelyopterus lucenai). The occurrence of these species in the BHRT probably resulted from accidental escapes from fish farm tanks in the nearby localities, as well as due to floods in the rice farms during the rainy season. The introduction of these non-native fishes represents a potential threat to aquatic ecosystems in the region, and may cause serious damage to the local diversity and artisanal fisheries. Therefore, it is essential to map all fish farming activities in the BHRT and to regulate and supervise these activities. In order to promote the use of native fish species and reduce the chance of introduction of invasive species is also important to enhance the technical assistance to the local fish farmers.

Keywords: Non-native Species, Allochthonous Species, Alien Species, Invasive Species, Tramandaí River Drainage.

Recebido em: 25 de novembro de 2015 Aprovado em: 29 de dezembro de 2015

\section{INTRODUÇÃO}

A introdução de espécies exóticas é considerada atualmente uma das principais ameaças à biodiversidade (CLAVERO; GARCÍA-BERTHOU, 2005). Espécies de peixes não-nativas podem causar sérios danos aos ecossistemas naturais, especialmente a partir da 
introdução de patógenos e parasitos, degradação de hábitats, competição por recursos, hibridização e predação de espécies nativas (CUCHEROUSSET; OLDEN, 2011). Além dos impactos ambientais, espécies invasoras podem ocasionar sérios prejuízos socioeconômicos, em função da redução dos estoques de peixes nativos e consequente diminuição nas capturas das espécies alvo das atividades pesqueiras (AGOSTINHO; JULIO JÚNIOR, 1996; QUEROL et al., 2005; PERES; KLIPPEL, 2008; CUCHEROUSSET; OLDEN, 2011). Esta questão é particularmente importante em regiões como o Estado do Rio Grande do Sul, no sul do Brasil, onde cerca de $47 \%$ da pesca é representada por pescadores artesanais de pequena escala que utilizam esta atividade como um recurso importante para a subsistência (PERES; KLIPPEL, 2008).

Nas últimas décadas, no Rio Grande do Sul, tem ocorrido um aumento significativo de registros de peixes não-nativos em ambientes naturais, incluindo a presença de tilápia (Tilapia sp.) na bacia hidrográfica do rio Mampituba, no município de Torres (RAMOS; VIEIRA, 2001) e da carpa-húngara (Cyprinus carpio) na bacia do médio rio Uruguai (QUEROL et al. 2005). No sistema da laguna dos Patos, foram também documentadas diversas espécies de peixes exóticos, como o bagre-norte-africano (Clarias gariepinus) (BRAUN; MILANI; FONTOURA, 2003) e quatro espécies de carpas (Ctenopharyngodon idella, C. carpio, Hypophthalmichthys molitrix, $H$. nobilis) (GARCIAS et al., 2004; MILANI; FONTOURA, 2007), além de três espécies alóctones provenientes da bacia do rio Uruguai: a maria-luiza (Pachyurus bonariensis) (PINTO et al., 2001; BRAUN, 2005; DUFECH; FIALHO, 2006; MILANI; FONTOURA, 2007; MARQUES, 2007), o peixe-cachorro (Acestrorhynchus pantaneiro) (SACCOL-PEREIRA; MILANI; FIALHO, 2006), e o porrudo (Trachelyopterus lucenai) (BERTOLETTI; PEZZI; PEREIRA, 1995; BRAUN, 2005; MARQUES, 2007). No rio dos Sinos, que pertence ao sistema da laguna dos Patos, já foram registradas 12 espécies invasoras de peixes (LEAL, BREMM; SCHULZ, 2009). Na bacia hidrográfica do rio Tramandaí (BHRT), foram reportadas recentemente as ocorrências do porrudo (ARTIOLI; MAIA, 2010), do peixe-cachorro (ARTIOLI et al., 2013), da carpa-húngara e da tilápiado-Nilo (Oreochromis niloticus) (GUIMARÃES, 2009).

A pesca artesanal na BHRT é uma importante atividade socioeconômica para as comunidades locais (SILVA; PERES, 2005), sendo estimado que cerca de 1500 famílias dependam exclusivamente desta atividade na região (PERES; CAMPANI; KLIPPEL, 2005; SILVA, PERES, 2005). O sistema estuarino-lagunar de Tramandaí (SELT) é a principal área de pesca de toda a bacia hidrográfica, com uma grande variedade de artes de pesca utilizadas (SILVA; PERES, 2005), sendo a pesca do bagre (Genidens spp.) a principal atividade pesqueira na localidade (MACHADO et al., 2010, 2012).
No presente trabalho são apresentados novos registros de peixes não-nativos no SELT, assim como uma revisão das espécies invasoras documentadas na BHRT. Além disso, são discutidas possíveis implicações para as atividades pesqueiras locais e considerações em relação à legislação atual referente à piscicultura de espécies de peixes não-nativas no Estado do Rio Grande do Sul.

\section{MATERIAIS E MÉTODOS \\ 2.1 Área de Estudo}

A BHRT é uma das três principais bacias hidrográficas do Rio Grande do Sul (REIS et al., 2003; MILANI; FONTOURA 2007), sendo formada por 41 lagoas que se comunicam com o SELT por meio do rio Tramandaí e do canal Camarão, que desaguam respectivamente que na laguna Tramandaí e na laguna Armazém (GUIMARÃES, 2013). Por sua vez, o SELT (ca. $29^{\circ} 59^{\prime} \mathrm{S}$; $50^{\circ} 08^{\prime} \mathrm{W}$ ) é formado por duas lagunas (Tramandaí e Armazém) que se comunicam com o Oceano Atlântico através de um estreito canal de cerca de 1,5 km de extensão (Figura 1). A temperatura superficial da água e a salinidade no estuário podem variar de 12 a $29^{\circ} \mathrm{C}$ e de 1,77 a 37,2 , respectivamente, dependendo da maré e do influxo de água do rio Tramandaí (SCHWARZBOLD; SCHÄFER, 1984; ROCHA, 2013).

As informações mais recentes apontam para cerca de 100 espécies de peixes de água doce na BHRT, incluindo espécies registradas no conjunto de lagoas costeiras e também aquelas presentes nos rios Maquiné e Três Forquilhas (MALABARBA et al., 2013). Dentre elas, o lambari-escuro (Astyanax douradilho), o cascudinho-listrado (Epactionotus bilineatus), o caráde-lagoa (Gymnogeophagus lacustris), a viola-da-lagoa (Rineloricaria quadrensis) e três espécies de peixe-rei (Odontesthes bicudo, $O$. ledae, $O$. piquava) são consideradas endêmicas da BHRT (FERRER; DONIN; MALABARBA, 2015).

\subsection{Coleta e Análise de Dados}

Os dados foram obtidos por meio de entrevistas informais com pescadores e confirmados a partir do resgate de fotografias tanto pessoais, quanto publicadas em um sítio eletrônico voltado à pesca da região, no período entre 2007 e 2008. Paralelamente aos registros confirmados por meio de fotografias, realizou-se um levantamento bibliográfico sobre as espécies de peixes não-nativos ocorrentes na BHRT.

Em abril de 2008, foi realizada uma pesquisa junto à Fundação Estadual de Proteção Ambiental Henrique Luiz Roesseler (FUNDAÇÃO ESTADUAL DE PROTEÇÃO AMBIENTAL HENRIQUE LUIZ ROESSELER, 2008) visando verificar quantos empreendimentos de piscicultura de espécies exóticas haviam licenciados na região. Além disso, foram realizadas entrevistas por telefone e correio eletrônico com os escritórios regionais da Associação Riograndense de Empreendimentos de Assistência Técnica e Extensão Rural (EMATER), bem como os 
escritórios das secretarias municipais de agricultura, pesca e meio ambiente para identificar quais municípios da BHRT possuíam atividades de piscicultura.

Por fim, foi avaliada a classificação das espécies registradas segundo a mais recente legislação do Estado do Rio Grande do Sul referente às normas e controle de espécies exóticas invasoras (PORTO ALEGRE, 2013). Nesse sentido, as espécies listadas na Categoria 1 desta normativa teriam "proibido seu transporte, criação, soltura ou translocação, cultivo, propagação (por qualquer forma de reprodução), comércio, doação ou aquisição intencional sob qualquer forma", enquanto as da Categoria 2 "poderiam ser utilizadas em condições controladas, com restrições, sujeitas à regulamentação específica". Com respeito ainda à categoria 2, a mesma normativa menciona que "compete ao órgão licenciador permitir o cultivo ou a criação de espécies exóticas invasoras mediante autorização específica, sujeitas a Análise de Risco e Plano de Controle Ambiental".

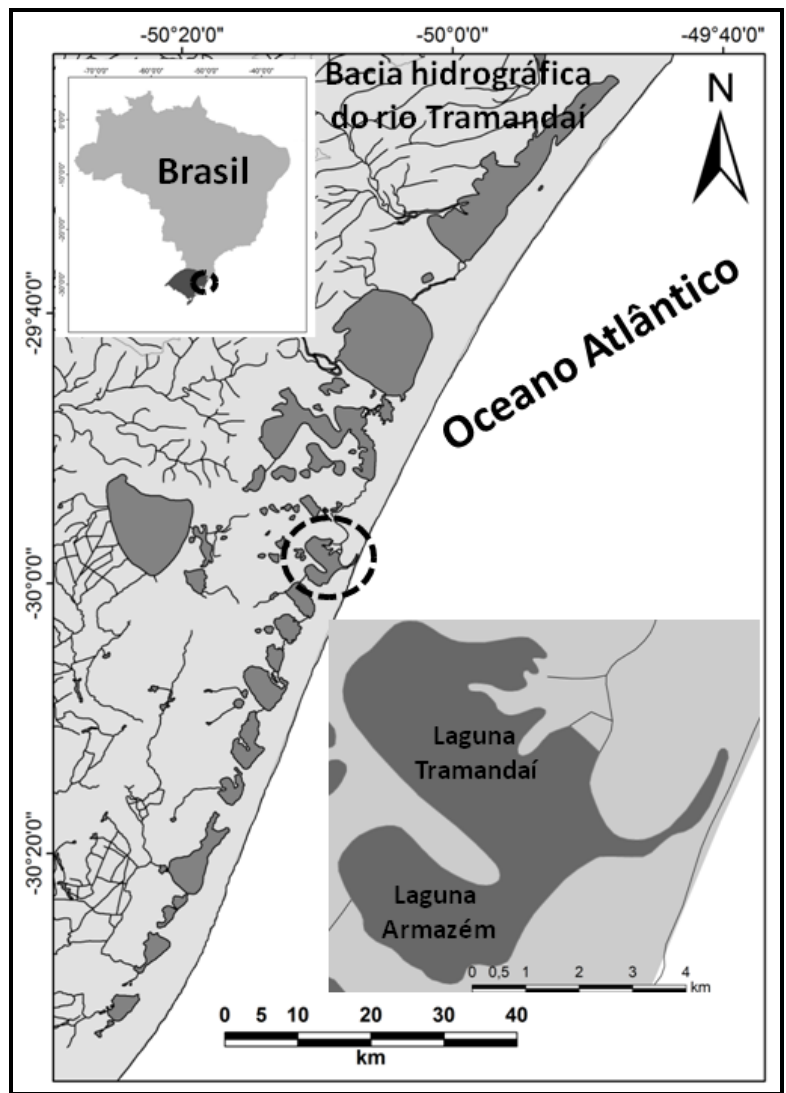

Figura 1 - Bacia hidrográfica do rio Tramandaí (BHRT), litoral norte do Rio Grande do Sul, Brasil. Em destaque o sistema estuarino-lagunar de Tramandaí (SELT) formado pelas lagunas Tramandaí e Armazém, onde foram documentadas as espécies não-nativas. Fonte: Carvalho (2015).

\section{RESULTADOS E DISCUSSÃO}

Com base nas fotografias obtidas, foi possível documentar a captura de oito exemplares de peixes nãonativos no SELT e águas adjacentes no período entre 2007 e 2008, incluindo dois espécimes de carpa-cabeçagrande (H. nobilis) (Figura 2: A e B), dois de carpa- prateada (H. molitrix) (Figura 2: C e D), dois de carpacapim (C. idella) (Figura 2: E e F), e um de carpahúngara (C. carpio) (Figura 2: F). Em abril de 2007, também foi registrada a captura de um exemplar da família Serrasalmidae, possivelmente um pacu (cf. Piaractus mesopotamicus). Entretanto, a fotografia disponível não permitiu a identificação segura deste último exemplar (Figura 2: G), especialmente em função da crescente produção de híbridos no Brasil entre espécies da família Serrasalmidae ( $C$. macropomum, Piaractus mesopotamicus e $P$. brachypomus) (e.g. HASHIMOTO et al., 2012).

Segundo informações fornecidas pelos pescadores, todos os exemplares foram capturados com rede de espera na laguna do SELT, com tarrafa ou coca na foz do estuário e na área marinha adjacente. Os pescadores relataram ainda que os espécimes de carpas capturados na foz do estuário apresentavam sinais claros de debilitação, possivelmente em função da salinidade, podendo, em alguns casos, ser capturados manualmente.

De acordo com as fotografias obtidas e a revisão da literatura, existem registros comprovados de nove espécies não-nativas de peixes na BHRT, sendo quatro espécies da família Cyprinidae (C. idella, C. carpio, $H$. nobilis, $H$. molitrix), além de representantes das famílias Anostomidae (Leporinus obtusidens), Auchenipteridae (T. lucenai), Characidae (A. pantaneiro), Cichlidae (O. niloticus) e Serrasalmidae (cf. P. mesopotamicus). Esses registros incluem tanto informações prévias existentes na literatura, quanto espécies que estão sendo documentadas pela primeira vez na região (Tabela 1 ).

Conforme alguns autores (e.g. LEAL et al., 2010) e a própria legislação brasileira (e.g. INSTITUTO BRASILEIRO DO MEIO AMBIENTE E AMAZÔNIA LEGAL, 1998; CONSELHO NACIONAL DO MEIO AMBIENTE, 2009), as espécies de peixes não-nativas podem ser classificadas em duas categorias, de acordo com o local de origem: espécies alóctones (i.e. nativas do Brasil, mas provenientes de outras bacias hidrográficas) e espécies exóticas (i.e. proveniente de outros continentes). No caso das espécies registradas na BHRT, enquadram-se na primeira categoria o peixecachorro (A. pantaneiro), a piava (L. obtusidens), o porrudo (T. lucenai) e o pacu (cf. Piaractus mesopotamicus) por serem espécies oriundas de outras bacias hidrográficas brasileiras (e.g. bacias dos rios Uruguai e Paraná), enquanto na segunda, as carpas ( $C$. idella, C. carpio, H. molitrix e H. nobilis) e a tilápia ( $O$. niloticus) (Tabela 1). Contudo, do ponto de vista ecológico, é importante destacar que o fato de espécies serem provenientes de outras bacias brasileiras não significa que elas sejam necessariamente menos impactantes que aquelas oriundas de outros continentes (AGOSTINHO; PELICICE; JÚLIO JUNIOR, 2006; VITULE, 2009). Desta forma, é fundamental que existam medidas efetivas de controle que reduzam a probabilidade de introdução de ambos os grupos de espécies.

Em relação a mais recente legislação do Estado do Rio Grande do Sul, referente ao controle de espécies 
exóticas invasoras (PORTO ALEGRE, 2013; 2014), as três espécies oriundas da bacia do rio Uruguai ( $A$. pantaneiro, cf. P. mesopotamicus e T. lucenai) estão incluídas na Categoria 1, que proíbe o cultivo/propagação, enquanto $C$. idella, C. carpio, $H$. molitrix e $H$. nobilis e $O$. niloticus estão na Categoria 2, que permite cultivo/criação, mas exige autorização específica do órgão ambiental (Tabela 1). Do ponto de vista de conservação, é importante ainda destacar que $C$. carpio foi classificada entre as 100 espécies invasoras mais perigosas do mundo (LOWE; BROWNE; BOUDJELAS, 2000). Essa espécie, assim como as demais da família Cyprinidae, foi introduzida para fins de piscicultura no Rio Grande do Sul, especialmente na década de 1970 (POLI; GRUMANN; BORGHETTI, 2000).
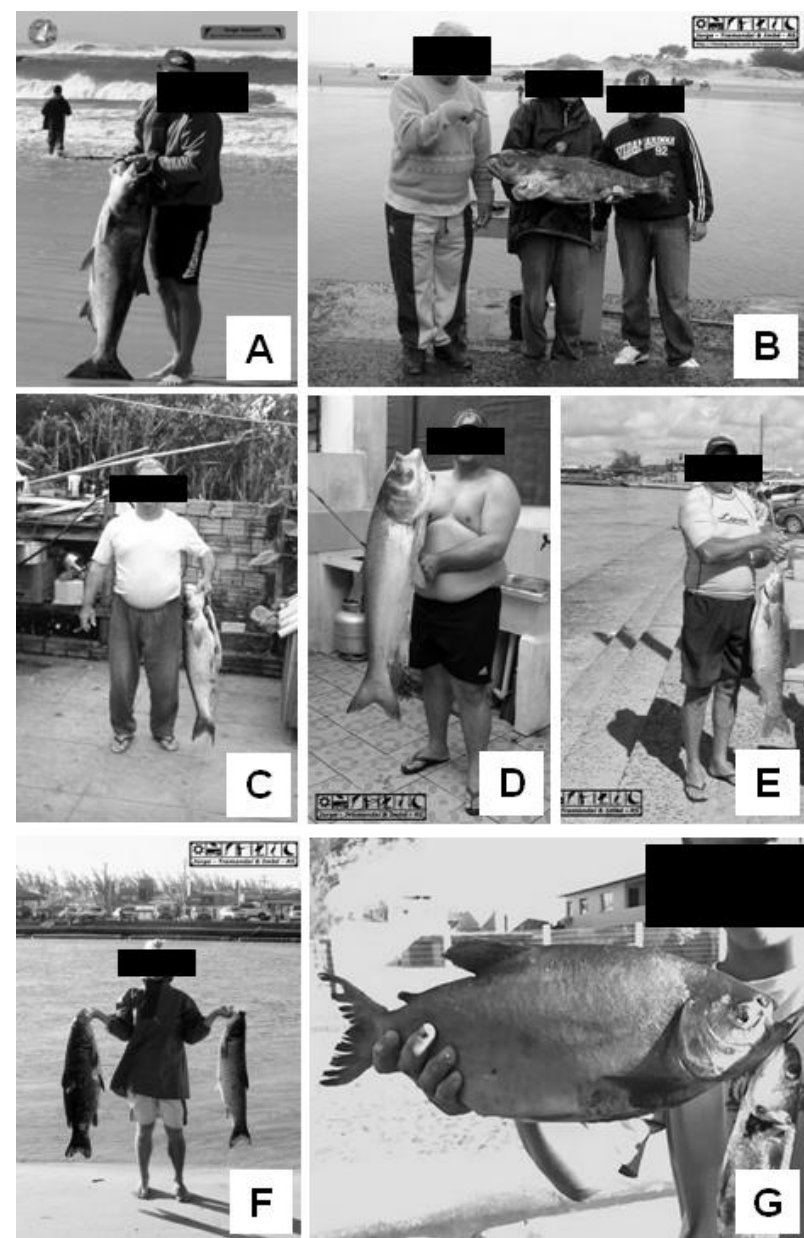

Figura 2 - Espécimes de peixes não-nativos capturados por pescadores artesanais no sistema estuarino-lagunar de Tramandaí, litoral norte do Rio Grande do Sul entre 2007 e 2008: carpa-cabeça-grande (Hypophthalmichthys nobilis) (A e B), carpa-prateada (H. molitrix) (C e D), carpa-capim (Ctenopharyngodon idella) (E e F lado esquerdo do pescador), carpa-húngara (Cyprinus carpio) (F, lado direito do pescador) e pacu (cf. Piaractus mesopotamicus) (G).

No Brasil, igualmente como em outras regiões do mundo, a aquicultura é a principal fonte de introdução de espécies não-nativas de peixes de água doce (NAYLOR; WILLIAMS; $\quad$ STRONG, 2001,
AGOSTINHO; PELICICE; JÚLIO JÚNIOR. 2006; LIMA-JUNIOR et al., 2012). Conforme os dados cadastrais obtidos na FEPAM (FUNDAÇÃO ESTADUAL DE PROTEÇÃO AMBIENTAL HENRIQUE LUIZ ROESSELER, 2008), foram identificados somente dois empreendimentos com atividades licenciadas de piscicultura de espécies exóticas nos municípios da BHRT (Osório e Terra da Areia). Entretanto, a partir das entrevistas realizadas com técnicos das prefeituras e EMATER, foi constatado que, pelo menos, 14 municípios que integram a BHRT desenvolvem atividades de piscicultura, provavelmente também incluindo criação de espécies não-nativas. Assim, a origem dos exemplares registrados neste estudo provavelmente está relacionada ao escape acidental de indivíduos de tanques de piscicultura da região, possivelmente em períodos de chuvas mais intensas. No caso das espécies consideradas alóctones, alguns autores sugerem que a ocorrência e dispersão dessas espécies na BHRT poderiam ser resultantes da própria geomorfologia da planície costeira, facilitadas por atividades relacionadas à irrigação das lavouras de arroz e períodos de chuvas intensas (ARTIOLI; MAIA, 2010; ARTIOLI et al., 2013).

Embora o estabelecimento de algumas das espécies exóticas registradas no SELT, como as carpas, possa ser limitado pela influência salina do Oceano Atlântico, é provável que a dispersão desses peixes esteja também ocorrendo para outras áreas com condições mais apropriadas na BHRT, tais como as lagoas costeiras e outros corpos de água doce. O estabelecimento de espécies de peixes não-nativos tem sido documentado em outras bacias hidrográficas do Estado do Rio Grande do Sul. Na bacia do médio rio Uruguai, a ocorrência em ambiente natural de fêmeas maduras de $C$. carpio, aptas à reprodução, sugere que a espécie já poderia estar estabelecida na região (QUEROL et al., 2005). Similarmente, o registro de atividade reprodutiva de $P$. bonariensis e $T$. lucenai no sistema da laguna dos Patos indica que estas espécies também podem estar estabelecidas nesta bacia (BRAUN, 2005; MARQUES 2007). Segundo MILANI; FONTOURA (2007), P. bonariensis é capturada em escala comercial na lagoa do Casamento, sistema da laguna dos Patos, onde ocorrem também capturas e comercialização de carpas (C. idella e/ou C . carpio). Os autores também sugerem que além do $P$. bonariensis, as duas espécies de carpas estariam estabelecidas nesta localidade.

Embora o estabelecimento de algumas das espécies exóticas registradas no SELT, como as carpas, possa ser limitado pela influência salina do Oceano Atlântico, é provável que a dispersão desses peixes esteja também ocorrendo para outras áreas com condições mais apropriadas na BHRT, tais como as lagoas costeiras e outros corpos de água doce. O estabelecimento de espécies de peixes não-nativos tem sido documentado em outras bacias hidrográficas do Estado do Rio Grande do Sul. Na bacia do médio rio Uruguai, a ocorrência em ambiente natural de fêmeas 
maduras de $C$. carpio, aptas à reprodução, sugere que a espécie já poderia estar estabelecida na região (QUEROL et al., 2005). Similarmente, o registro de atividade reprodutiva de $P$. bonariensis e $T$. lucenai no sistema da laguna dos Patos indica que estas espécies também podem estar estabelecidas nesta bacia (BRAUN, 2005; MARQUES 2007). Segundo MILANI; FONTOURA (2007), P. bonariensis é capturada em escala comercial na lagoa do Casamento, sistema da laguna dos Patos, onde ocorrem também capturas e comercialização de carpas (C. idella e/ou C. carpio). Os autores também sugerem que além do $P$. bonariensis, as duas espécies de carpas estariam estabelecidas nesta localidade.

Embora o estabelecimento de algumas das espécies exóticas registradas no SELT, como as carpas, possa ser limitado pela influência salina do Oceano Atlântico, é provável que a dispersão desses peixes esteja também ocorrendo para outras áreas com condições mais apropriadas na BHRT, tais como as lagoas costeiras e outros corpos de água doce. $\mathrm{O}$ estabelecimento de espécies de peixes não-nativos tem sido documentado em outras bacias hidrográficas do Estado do Rio Grande do Sul. Na bacia do médio rio Uruguai, a ocorrência em ambiente natural de fêmeas maduras de C. carpio, aptas à reprodução, sugere que a espécie já poderia estar estabelecida na região (QUEROL et al., 2005). Similarmente, o registro de atividade reprodutiva de $P$. bonariensis e $T$. lucenai no sistema da laguna dos Patos indica que estas espécies também podem estar estabelecidas nesta bacia (BRAUN, 2005; MARQUES 2007). Segundo MILANI; FONTOURA (2007), P. bonariensis é capturada em escala comercial na lagoa do Casamento, sistema da laguna dos Patos, onde ocorrem também capturas e comercialização de carpas (C. idella e/ou C. carpio). Os autores também sugerem que além do $P$. bonariensis, as duas espécies de carpas estariam estabelecidas nesta localidade.

No caso da BHRT, enquanto os registros de carpas e tilápias parecem ser relativamente pontuais, $A$. pantaneiro, L. obtusidens e, especialmente, T. lucenai têm sido frequentemente reportadas em diferentes localidades do litoral norte do Rio Grande do Sul (ARTIOLI; MAIA, 2010; ARTIOLI et al., 2013, MALABARBA et al., 2013; ROCHA; HARTZ, 2013), indicando que esta última espécie está bem estabelecida na região. Contudo, não há informações sobre os prejuízos que estes peixes alóctones poderiam estar trazendo para o ambiente, bem como para a comunidade pesqueira local. Considerando o panorama atual, há um sério risco das espécies exóticas de carpas e tilápias também se estabelecerem na BHRT. Embora, por um lado, os pescadores possam utilizar essas espécies nãonativas como recurso alternativo na pesca artesanal, a introdução destas espécies pode acarretar danos a médio e longo prazo para os estoques de peixes nativos e para todo o ecossistema (QUEROL et al., 2005; PERES; KLIPPEL, 2008).

Tabela 1 - Espécies de peixes não-nativas registradas na Bacia do Rio Tramandaí (BHRT).

\begin{tabular}{|c|c|c|c|c|c|c|}
\hline Familia & Táxon & $\begin{array}{l}\text { Nome Comum } \\
\text { (English name) }\end{array}$ & $\begin{array}{l}\text { Ano de } \\
\text { registro }\end{array}$ & Fontes & $\begin{array}{l}\text { Classificação baseada } \\
\text { na bacia de origem }\end{array}$ & $\begin{array}{l}\text { Portaria SEMA no. } 79 \\
\text { de } 31 / 10 / 2013\end{array}$ \\
\hline Anostomidae & $\begin{array}{c}\text { Leporinus obtusidens } \\
\text { (VALENCIENNES, 1837) }\end{array}$ & Piava & $2009-2013$ & $\begin{array}{l}\text { ROCHA; HARTZ 2013; } \\
\text { GUIMARÃES, } 2013\end{array}$ & Alóctone & Não consta \\
\hline \multirow{3}{*}{ Auchenipteridae } & Trachelyopterus lucenai & & & $\begin{array}{l}\text { GUIMARÃES, 2009, } \\
\text { ARTIOLI; MAIA, 2010; }\end{array}$ & \multirow{3}{*}{ Alóctone } & \multirow{3}{*}{ Lista A (Anexo 3) } \\
\hline & $\begin{array}{l}\text { BERTOLETTI; PEZZI; } \\
\text { PEREIRA, } 1995\end{array}$ & Porrudo, Penharol & 2010 & ROCHA; HARTZ 2013; & & \\
\hline & & & & MAL_ABARBA et al., 2013 & & \\
\hline Cichlidae & $\begin{array}{l}\text { Oreochromis niloticus } \\
\text { (LINNAEUS, 1758) }\end{array}$ & $\begin{array}{l}\text { Tilápia-do-nilo } \\
\text { (Nile tilapia) }\end{array}$ & 1999,2009 & $\begin{array}{l}\text { RAMOS; VIEIRA, 2001; } \\
\text { GUIMARÃES, } 2009\end{array}$ & Exótica & $\begin{array}{c}\text { Categoria } 2 \\
\text { Lista A (Anexo 3) }\end{array}$ \\
\hline Characidac & $\begin{array}{l}\text { Acestrorhynchus pantaneiro } \\
\text { MENEZES, } 1992\end{array}$ & Peixe-cachorro & 2011 & $\begin{array}{c}\text { ARTIOLI et al. 2013; } \\
\text { ROCHA; HARTZ 2013; } \\
\text { MALABARBA et al., } 2013\end{array}$ & Alóctone & $\begin{array}{c}\text { Categoria } 1 \\
\text { Lista A (Anexo 3) }\end{array}$ \\
\hline \multirow{4}{*}{ Cyprinidae } & $\begin{array}{l}\text { Hypophthalmichthys nobilis } \\
\text { (RICHARDSON, 1845) }\end{array}$ & $\begin{array}{l}\text { Carpa-cabeça-grande } \\
\text { (Bighead carp) }\end{array}$ & 2007 & Este estudo (Figura $2 \mathrm{~A} \mathrm{e} \mathrm{B}$ ) & Exótica & $\begin{array}{c}\text { Categoria } 2 \\
\text { Lista A (Anexo 3) }\end{array}$ \\
\hline & $\begin{array}{l}\text { Hypophthalmichthys molitrix } \\
\text { (VALENCIENNES, 1844) }\end{array}$ & $\begin{array}{l}\text { Carpa-prateada } \\
\text { (Silver carp) }\end{array}$ & $\begin{array}{l}2004-2005 \\
2007\end{array}$ & Este estudo (Figura $2 \mathrm{C}$ e D) & Exótica & $\begin{array}{c}\text { Categoria } 2 \\
\text { Lista A (Anexo 3) }\end{array}$ \\
\hline & $\begin{array}{l}\text { Ctenopharyngodon idella } \\
\text { (VALENCIENNES, 1844) }\end{array}$ & $\begin{array}{l}\text { Carpa-capim } \\
\text { (Grass carp) }\end{array}$ & 2007 & Este estudo (Figura 2 E e F) & Exótica & $\begin{array}{c}\text { Categoria } 2 \\
\text { Lista A (Anexo 3) }\end{array}$ \\
\hline & $\begin{array}{c}\text { Cyprinus carpio } \\
\text { LINNAEUS, } 1758\end{array}$ & $\begin{array}{l}\text { Carpa-húngara ou } \\
\text { Carpa-comum } \\
\text { (Common carp) }\end{array}$ & 2007,2009 & $\begin{array}{l}\text { Este estudo (Figura 2 F); } \\
\text { GUIMARÂES, 2009 }\end{array}$ & Exótica & $\begin{array}{c}\text { Categoria } 2 \\
\text { Lista A (Anexo 3) }\end{array}$ \\
\hline Serrasalmidae & $\begin{array}{l}\text { cf. Piaractus mesopotamicus * } \\
\text { (HOLMBERG, 1887) }\end{array}$ & Pacu & 2007 & Este estudo (Figura 2 G) & Alóctone & $\begin{array}{c}\text { Categoria } 2 \\
\text { Lista A (Anexo 3) }\end{array}$ \\
\hline
\end{tabular}

* O exemplar capturado pode ser também um híbrido.

\section{CONCLUSÃO}

Considerando tanto a existência de uma legislação específica no Estado do Rio Grande do Sul para uso e manejo de peixes exóticos, quanto o impacto negativo que estas espécies podem ter sobre a pesca artesanal e a diversidade local, é fundamental que seja realizado um mapeamento completo dos empreendimentos de piscicultura na BHRT, acompanhado de uma regularização e fiscalização das atividades existentes. Ao mesmo tempo, há a necessidade do aumento de atividades de extensão e assistência técnica para os piscicultores, com vistas a incentivar o manejo de espécies nativas, bem como reduzir a probabilidade de 
escape de espécies exóticas aos ambientes naturais. Concomitantemente se faz necessário um monitoramento de longo prazo das comunidades ícticas do BHRT, só assim poderemos avaliar qual é a real influência das espécies invasoras sobre as nativas.

\section{AGRADECIMENTOS}

Gostaríamos de agradecer aos pescadores Agenor Costa, Artur Lima Sala e ao fotógrafo Jorge Humberto Muniz Goulart pelo fornecimento das fotos e informações. A Lucas Milmann de Carvalho pela confecção do mapa.

\section{REFERÊNCIAS}

AGOSTINHO, A.A.; JULIO JÚNIOR, H.F. Peixes de outras águas. Ciência Hoje, Rio de janeiro, v. 21, n. 124, p. 36-44, 1996.

AGOSTINHO, A.A.; PELICICE, F.M.; JÚLIO JÚNIOR, H.F. Biodiversidade e introdução de espécies de peixes: unidades de conservação. In: CAMPOS, J.B.; TOSSULINO, M.G.P.; MÜLLER, C.R.C. Unidades de conservação: ações para valorização da biodiversidade. Curitiba: Instituto Ambiental do Paraná, 2006. p. 95-117.

ARTIOLI, L.G.S.; MAIA, R. Pisces, Siluriformes, Auchnipteridae, Trachelyopterus lucenai Bertoletti, Pezzi da Silva \& Pereira, 1995: historical occurence and distribution extension. Check List, São Paulo, v. 6, n. 4, p. 515-516, oct. 2010. Disponível em: < http://www.checklist.org.br/getpdf?NGD023-10>. Acesso em: 9 nov. 2015.

ARTIOLI, L.G.S. et al. First record of the non-native species Acestrorhynchus pantaneiro Menezes, 1992 (Characiformes, Acestrorhynchidae) in the Tramandaí River System, Rio Grande do Sul, Brazil. Pan-American Journal of Aquatic Sciences, v.8, n.1, p. 51-54. mar. 2013. Disponível em: http://www.panamjas.org/pdf_artigos/PANAMJAS_8(1)_5 1-54.pdf >. Acesso em: 9 nov. 2015.

BERTOLETTI, J.J.; PEZZI, J.F.; PEREIRA, E.H.L. A new species of the catfish genus Trachelyopterus (Siluriformes, Auchenipteridae) from Southern Brazil. Revue Française d'Aquariologie, Nancy, v.22, n. 3-4, p. 71-74. 1995.

BRAUN, A.S.; MILANI, P.C.C.; FONTOURA, N.F. Registro da introdução de Clarias gariepinus (Siluriformes, Clariidae) na laguna dos Patos. Biociências, Porto Alegre, v.11 n.1, p. 101-102, jun. 2003.

BRAUN, A.S. Biologia reprodutiva e identificação do uso de lagoa marginal como sítio de reprodução para espécies dominantes da ictiofauna da lagoa do Casamento, sistema nordeste da laguna dos Patos, RS. 2005. 145 f. Tese (Doutorado em Zoologia) - Pontifícia Universidade Católica do Rio Grande do Sul, Porto Alegre, 2005.

CLAVERO, M.; GARCÍA-BERTHOU E. Invasive species are leading cause of animal extinctions. Trends in Ecology \& Evolution, Girona, v. 20, n. 3, p. 110, jan. 2005. doi:10.1016/j.tree.2005.01.003.

CONSELHO NACIONAL DO MEIO AMBIENTE. Resolução $n^{\circ}$ 413, de 26 de junho de 2009. Dispõe sobre o licenciamento ambiental da aqüicultura e dá outras providências. Diário Oficial da União, Brasília, 30 de junho de 2009.

CUCHEROUSSET J.; OLDEN J.D. Ecological impacts of non-native freshwater fishes. Fisheries, v. 36, n. 5, p.215230, may 2011.

DUFECH, A.P.S.; FIALHO, C.B. Biologia populacional de Pachyurus bonariensis Steindachner, 1878 (Perciformes, Sciaenidae), uma espécie alóctone no sistema hidrográfico da laguna dos patos, Brasil. Biota Neotropical, Campinas, v.7, n.1, p. 105-110, mar. 2006.

$\begin{array}{lrrr}\text { FUNDAÇÃO } & \text { ESTADUAL } & \text { DE } & \text { PROTEÇÃO } \\ \text { AMBIENTAL HENRIQUE } & \text { LUIZ } & \text { ROESSELER. } \\ \text { Agrossilvipastoril. } & 2008 . & \text { Disponível em: } \\ \text { <http://www.fepam.rs.gov.br/licenciamento/area3/default.a } \\ \text { sp>. Acesso em: } 28 \text { abr. 2008. }\end{array}$

FERRER, J; DONIN, L.M; MALABARBA, L.R. A new species of Ituglanis Costa \& Bockmann, 1993 (Siluriformes: Trichomycteridae) endemic to the Tramandaí-Mampituba ecoregion, southern Brazil. Zootaxa, v. 4020, n. 2, p. 375-389, sep. 2015.

GARCIA, A.M. et al. First records of introduced carps (Teleostei, Cyprinidae) in the natural habitats of Mirim and Patos Lagoon estuary, Rio Grande do Sul, Brazil. Revista Brasileira de Zoologia, Curitiba, v. 21, n. 1, p. 157-159, mar. 2004.

GUIMARÃES, T. F. R. Conectividade e padrões de riqueza de espécies de peixes nas lagoas do litoral norte do Rio Grande do Sul. 2009. Monografia (Graduação) Universidade Federal do Rio Grande do Sul, Porto Alegre, 2009.

GUIMARÃES, T.F.R. Influência de variáveis de paisagem sobre a riqueza e composição de assembleias de peixes em lagoas costeiras no sul do Brasil. 2013. 90 f. Dissertação (Mestrado Biologia Animal) - Universidade Federal do Rio Grande do Sul, Porto Alegre, 2013.

HASHIMOTO, D.T. et al. Interspecific fish hybrids in Brazil: management of genetic resources for sustainable use. Reviews in Aquaculture, v. 4, n. 2, p. 108-118, jun. 2012.

INSTITUTO BRASILEIRO DO MEIO AMBIENTE E AMAZÔNIA LEGAL. Portaria $n^{\circ}$. 145-N, de 29 de outubro de 1998. Estabelece normas para a introdução, reintrodução e transferência de peixes, crustáceos, moluscos, e macrófitas aquáticas para fins de aqüicultura, excluindo-se as espécies animais ornamentais. Diário Oficial da União. Brasília, 29 de outubro de 1998.

LEAL, M.E.; BREMM, C.Q.; SCHULZ, U.H. Lista da ictiocenose da Bacia do Rio dos Sinos, sul do Brasil. Boletim do Instituto de Pesca, São Paulo, v. 35, n. 2, p. 307-317, 2009.

LIMA-JUNIOR, D.P. et al. Aquicultura, política e meio ambiente no Brasil: novas propostas e velhos equívocos. Natureza \& Conservação, v. 10, n. 1, p. 1-4. jul. 2012. 
LOWE, S.J.; BROWNE, M.; BOUDJELAS, S. 100 of the world's worst invasive alien species. Auckland: Invasive Species Specialist Group, 2000.

MACHADO, R. et al Pesca artesanal de bagres Ariidae na laguna Tramandaí, Rio Grande do Sul, Brasil. In: CONGRESSO BRASILEIRO DE OCEANOGRAFIA, 3. 2010, Rio Grande, Anais... Rio Grande. CD-ROM.

MACHADO, R. et al.. Occurrence of the marine catfish Genidens machadoi (Siluriformes, Ariidae) in the Tramandaí lagoon, southern Brazil. Neotropical Biology and Conservation, São Leopoldo, v. 7, n. 3, p. 214-219, set./dez. 2012.

MALABARBA, L.R. et al. Guia de identificação dos peixes da bacia do rio Tramandaí. Porto Alegre: Via Sapiens, 2013

MARQUES, C.S. Estimativa de período reprodutivo e tamanho de primeira maturação de espécies dominantes da ictiofauna da lagoa dos Patos através do índice gonadossomático. 2007. 79 f. Dissertação (Mestrado em zoologia) - Pontifícia Universidade Católica do Rio Grande do Sul, Porto Alegre. 2007.

MILANI, P.C.C.; FONTOURA, N.F. Diagnóstico da pesca artesanal na lagoa do Casamento, sistema nordeste da laguna dos Patos: uma proposta do manejo. Biociências, Porto Alegre, v. 15, n. 1, p. 82-125. jan. 2007.

NAYLOR, R.L.; WILLIAMS, S.L.; STRONG, D.R. Aquaculture - a gateway for exotic species. Science, v. 294, n. 5547, p. 1655-1656. nov. 2001.

PERES, M. B.; CAMPANI, F.; KLIPPEL, S. O processo de gestão compartilhada da pesca artesanal da Bacia Hidrográfica do Rio Tramandaí e zona costeira adjacente, no nordeste do Rio Grande do Sul. In: ENCONTRO BRASILEIRO DE ICTIOLOGIA, 16., 2005, João PessoaPB. Anais... João Pessoa, 2005. p. 103.

PERES, M.B.; KLIPPEL, S. Impactos socioeconômicos da aqüicultura de espécies exóticas na pesca de pequena escala. 2008. Disponível em: <http://www.institutohorus.org.br/download/artigos/IGAR E_impacto_socioeconomico_da_introducao_de_spp_exotic as.pdf>. Acesso em 10 nov. 2015.

PINTO, R.F. et al. Primeiro registro de Pachyurus bonariensis (Steidachner, 1879) (Perciformes, Sciaenidae) para o sistema da laguna dos Patos, Rio Grande do Sul, Brasil. In: ENCONTRO BRASILEIRO DE ICTIOLOGIA, 14., 2001, São Leopoldo. Anais... São Leopoldo, 2001.

POLI, C.R.; GRUMANN, A.; BORGHETTI, J.R. Situação atual da aquicultura na região sul. In: VALENTI, W.C. et al. Aquicultura no Brasil: bases para um desenvolvimento sustentável. Brasília: CNPq; MCT, 2000. p. 323-352.
QUEROL, M.V.M. et al. Ocorrência da carpa húngara, Cyprinus carpio (Linnaeus, 1758) e disseminação parasitária, no arroio Felizardo, bacia do médio rio Uruguai, Uruguaiana, RS, Brasil. Biodiversidade Pampeana, Uruguaiana, v.3, p.21-23, dez. 2005.

RAMOS, L.; VIEIRA, J.P. Composição específica e abundância de peixes de zonas rasas dos cinco estuários do Rio Grande do Sul, Brasil. Boletim do Instituto de Pesca, São Paulo, v.27, n.1, p.109-121, 2001.

REIS, R.E. et al. Peixes In: FONTANA, C.S.; BENCKE, G.A.; REIS, R.E. Livro Vermelho das Espécies da Fauna Ameaçada de Extinção no Rio Grande do Sul. Porto Alegre: PUCRS, 2003.

ROCHA, C.M. Qualidade das águas na BH Rio Tramandaí. In: CASTRO D.; MELLO, R.S.P. Atlas Ambiental da Bacia Hidrográfica do Rio Tramandaí. Porto Alegre: Via Sapiens, 2013. p. 149-155.

ROCHA, E.A; HARTZ, S.M. Estabilidade e persistência de uma comunidade de peixes em lagoa costeira neotropical. Revista Brasileira de Biociências, Porto Alegre, v. 11, n. 2, p. 149-156, abr./jun. 2013

SACCOL-PEREIRA, A.; MILANI, P.C.C.; FIALHO, C.B. Primeiro registro de Acestrorhynchus pantaneiro Menezes, 1992 (Chariciformes, Acestrorhynchidae) no sistema da laguna dos Patos, Rio Grande do Sul, Brasil. Biota Neotropical, Campinas, v. 6, n. 3, set. 2006.

SCHWARZBOLD, A.; SCHÄFER, A. Gênese e morfologia das lagoas costeiras do Rio Grande do Sul, Brasil. Amazoniana, Manaus, v. 9, n. 1, p. 87-104. 1984.

PORTO ALEGRE. Secretaria do Meio Ambiente. Portaria Sema $n^{\circ}$ 79, de 31 de outubro de 2013. Reconhece a Lista de Espécies Exóticas Invasoras do Estado do Rio Grande do Sul e demais classificações, estabelece normas de controle e dá outras providências. Diário Oficial, Porto Alegre, 31 de outubro de 2013.

PORTO ALEGRE. Secretaria do Meio Ambiente. Instrução Normativa Sema $n^{\circ}$ 04, de 11 de novembro de 2014. Estabelece o ordenamento e controle das atividades que envolvem a criação de espécies de peixes exóticos invasores. Diário Oficial, Porto Alegre, 14 de novembro de 2014.

SILVA, E.B.; PERES, M.B. Análise preliminar do cadastro para o licenciamento ambiental da pesca artesanal no litoral norte do Rio Grande do Sul. In: CONGRESSO BRASILEIRO DE OCEANOGRAFIA, 2., 2005, Vitória, Anais. Vitoria, 2005. CD-ROM p. 1-3.

VITULE, J.R.S. Introduction of fishes in Brazilian continental ecosystems: Review, comments and suggestions for actions against the almost invisible enemy. Neotropical Biology and Conservation. v. 4, n. 2. p. 111122, may/aug. 2009 